\title{
Gender Difference in Brainstem Auditory Evoked Response among the Headphone Users
}

\author{
Latha R. ${ }^{1}$, Karthika Priyadharshini U. ${ }^{2}$, Jayamala A.K. ${ }^{2}$, Shyamala M. ${ }^{3}$ \\ ${ }^{1}$ Professor, ${ }^{2}$ Assistant Professor, Department of Physiology, ${ }^{3}$ MBBS Student, \\ Sri Venkateshwaraa Medical College Hospital and Research Centre, Ariyur, Puducherry, India
}

\begin{abstract}
About 5.6 billion people use mobile phones worldwide. India ranks second position with about 885 million users. Apart from its function of making calls, listening to music has become one of the popular functions, the sound delivered via headphones at high intensities. The purpose was to assess the possible deleterious effects of headphone usage on Brain stem Evoked Response Audiometry (BERA) in both genders. The study involved the recording of BERA using 30 volunteers between the age group of 18-21 years. They were divided into 2 groups. Group 1: Headphone users $<3$ hrs/day. Group 2: Headphone users $>3$ hrs/day. Both the groups comprised both genders. Data were compared by unpaired Student's $t$ test and one-way ANOVA. There was no significant difference in peak latencies of waves I to $\mathrm{V}$ and inter-peak latencies between genders on right and left ear of headphone users, except wave $V(p<0.01)$ on the left ear of headphone users with latency being more in males than in females. We conclude from the observation that prolonged headphone usage has a negative influence on brain stem auditory evoked potentials and there was no remarkable difference between the genders.
\end{abstract}

Keywords: Brain stem Evoked Response Audiometry, Gender difference, Head phone users, Mobile phones, Music.

\section{Introduction}

Headphone usage is drastically increased nowadays with about 173 million users, especially among teenagers ${ }^{1}$. The electromagnetic fields acquire importance due to the prevalence of symptoms like warmth, headache and fatigue ${ }^{2}$. Most of the students have cultivated a habit of listening to earphone music.

\section{Corresponding Author:}

Dr. R. Latha

Professor, Department of Physiology, Sri

Venkateshwaraa Medical College Hospital and

Research Centre, Ariyur, Puducherry-605 102, India

Office: 0413-2260601

Mobile: 9486419659

Fax: 0413-2644476

e-mail: lathaphysio@yahoo.co.in
This may lead to music-induced hearing loss ${ }^{3}$. Sound $\leq 85 \mathrm{~d} \mathrm{~B}$ is considered safe. The threshold value is $85 \mathrm{~d} \mathrm{~B}$, above which prolonged exposure to music for $\geq 8$ hours causes permanent hearing loss.

Brainstem Auditory Evoked Response or Brainstem Evoked Response Audiometry [BERA] is an objective way of eliciting brainstem potentials in response to audiological click stimuli. They are recorded by electrodes placed over the scalp. According to Jewett and Williston in 1971, the waves recorded were labeled as I to V. All the 5 waves are positive deflections that occur in first 10 milliseconds after the onset of auditory stimulus ${ }^{4}$. BERA is used to screen newborn's hearing ability, auditory threshold estimation, to determine the type and degree of hearing loss. It is also used in intra operative monitoring and to identify the lesions in the auditory nerve and the brainstem.

The advantage of analyzing the above topic is to create awareness among the teenagers about the 
deleterious effects of loud noise and electromagnetic waves emitted by the headphones. As little information is available on the influence of prolonged headphone usage on BERA, this study has been chosen to assess the possible deleterious effects of headphone usage on BERA in both genders.

\section{Material and Method}

An observational study was conducted among 30 volunteers aged between 18 and 21 years in the department of Physiology at Sri Venkateshwaraa Medical College Hospital and Research Centre in Puducherry, after obtaining Instituitional Ethical Committee (IEC) clearance before the commencement of study. After obtaining the prior consent from the subjects, they were divided into 2 groups. Group 1: Headphone users $<3 \mathrm{hrs} /$ day. Group 2: Headphone users $>3$ hrs/day. The subjects were selected by convenient sampling method and the selection was based on the following criteria. The subjects with history of systemic diseases like diabetes mellitus, hypertension and presbycusis were excluded. Informed written consent was obtained from all the subjects prior to the study. An ID code was assigned for the subjects to maintain confidentiality of the data obtained.

\section{Brainstem evoked response audiometry (BERA):} The pure Tone Audiometry (PTA) was done prior to the BERA recording in our hospital. PTA is a subjective assessment of hearing threshold, which is plotted on an audiogram displaying intensity as a function of frequency. Complete ENT examination including Rinne's test, Weber's test and absolute bone conduction test were carried out to rule out ear pathology. The BERA was recorded in our physiology research lab in the morning time at a pleasant temperature in a quite air conditioned room using Physiopac $\mathrm{PP}_{4}$, Medicaid Systems, Chandigarh. The subjects were asked to lie down on semi-reclined bed, made to relax completely in order to minimize the artefacts. Ornaments like earrings, hair clips were removed as it may alter the readings. Ground electrode was placed around the wrist. After cleaning the site with spirit (to prevent contact impedance) active electrode was placed on the respective mastoid of the ear through which the click stimuli has to be given. References electrode was kept in the vertex using cup electrode and the electrode paste. All the electrodes were plugged to the junction box. Contact impedance was constantly monitored using impedance meter to keep electrode impedance below $5 \mathrm{Kohms}$. The electrical activity of the brain stem was picked up by the electrodes when the click stimuli were given. The filtered, amplified, averaged values were displayed on the screen. The machine is provided with inbuilt artefact rejection facility 5 .

For recording the ABR 2000 click stimuli were given at $60 \mathrm{~d} B$ intensity. The rare type stimuli were generated by passing $0.1 \mathrm{~ms}$ square pulses through head phones. Monaural stimulation was used at the rate of 10 pulses per second and the contra lateral ear was masked with white noise with intensity less than that of the click stimuli. The responses in the first $10 \mathrm{~ms}$ were averaged. Filtration was done between $5 \mathrm{Htz}$ to $3000 \mathrm{Htz}$. The commonly seen waveforms are I, II, III, IV and V. These wave's latency, amplitude and duration were analyzed by the computer software - ABR - PHYSIOPAC $\mathrm{PP}_{4}$, MEDICAID SYSTEM, Chandigarh. Peak latencies of the waves I to V and inter-peak latencies I-V, I-III, III-V were analyzed. Both ears of each subject were taken as independent samples since anatomically both auditory pathways are different.

Statistical Analysis: The data were analyzed by using SPSS 17.0 software and expressed as mean \pm standard deviation. Student's ' $t$ ' test and one way ANOVA test were used to demonstrate the findings. The statistical probability of $\mathrm{P}<0.05$ was considered to be significant.

\section{Results}

Table 1: Right ear peak and inter-peak latencies of BERA among male and female head phone users

\begin{tabular}{|l|c|c|c|}
\hline PEAK LATENCY(ms) Mean \pm SD & Male & Female & P Value \\
\hline I & $1.53 \pm 0.27$ & $1.40 \pm 0.17$ & 0.28 \\
\hline II & $2.44 \pm 0.34$ & $2.42 \pm 0.29$ & 0.90 \\
\hline III & $3.50 \pm 0.25$ & $3.50 \pm 0.29$ & 0.99 \\
\hline IV & $4.51 \pm 0.37$ & $4.44 \pm 0.30$ & 0.70 \\
\hline V & $5.22 \pm 0.20$ & $5.29 \pm 0.29$ & 0.57 \\
\hline
\end{tabular}




\begin{tabular}{|l|c|c|c|}
\hline INTERPEAK LATENCY(ms) Mean \pm SD & Male & Female & P Value \\
\hline I-III & $1.96 \pm 0.27$ & $2.10 \pm 0.31$ & 0.38 \\
\hline I-V & $3.68 \pm 0.29$ & $3.76 \pm 0.15$ & 0.53 \\
\hline III-V & $1.71 \pm 0.32$ & $1.66 \pm 0.35$ & 0.77 \\
\hline
\end{tabular}

Table 1 shows that there was no significant difference in peak and inter- peak latencies between genders in right ear of head phone users

Table 2: Right ear peak and inter-peak latencies and of BERA among male and female head phone users

\begin{tabular}{|l|c|c|c|}
\hline PEAK LATENCY $(\mathbf{m s})$ Mean \pm SD & Male & Female & P Value \\
\hline I & $1.49 \pm 0.35$ & $1.42 \pm 0.24$ & 0.64 \\
\hline II & $2.39 \pm 0.30$ & $2.44 \pm 0.24$ & 0.75 \\
\hline III & $3.42 \pm 0.23$ & $3.61 \pm 0.20$ & 0.12 \\
\hline IV & $4.59 \pm 0.45$ & $4.43 \pm 0.35$ & 0.45 \\
\hline V & $5.15 \pm 0.14$ & $5.38 \pm 0.34$ & 0.19 \\
\hline INTERPEAK LATENCY(ms) Mean \pm SD & Male & Female & P Value \\
\hline I-III & $1.93 \pm 0.46$ & $2.19 \pm 0.24$ & 0.16 \\
\hline I-V & $3.66 \pm 0.37$ & $3.96 \pm 0.42$ & 0.20 \\
\hline III-V & $1.73 \pm 0.23$ & $1.77 \pm 0.45$ & 0.86 \\
\hline
\end{tabular}

Table 2 shows that there was no significant difference in peak and inter- peak latencies between genders in right ear of head phone users

Table 3: Left ear peak and inter-peak latencies and of BERA among male and female head phone users

\begin{tabular}{|l|c|c|c|}
\hline PEAK LATENCY $(\mathrm{ms})$ Mean \pm SD & Male & Female & P Value \\
\hline I & $1.62 \pm 0.21$ & $1.44 \pm 0.19$ & 0.09 \\
\hline II & $2.31 \pm 0.27$ & $2.46 \pm 0.38$ & 0.40 \\
\hline III & $3.61 \pm 0.38$ & $3.62 \pm 0.20$ & 0.94 \\
\hline IV & $4.40 \pm 0.38$ & $4.60 \pm 0.35$ & 0.31 \\
\hline V & $5.45 \pm 0.23$ & $5.11 \pm 0.09$ & $0.003 *$ \\
\hline INTERPEAK LATENCY(ms) Mean \pm SD & Male & Female & P Value \\
\hline I-III & $1.98 \pm 0.41$ & $2.19 \pm 0.31$ & 0.31 \\
\hline I-V & $3.82 \pm 0.34$ & $3.67 \pm 0.18$ & 0.30 \\
\hline III-V & $1.84 \pm 0.43$ & $1.48 \pm 0.23$ & 0.07 \\
\hline
\end{tabular}

Table 3 shows that there was a significant difference in peak latency of wave $\mathrm{V}(\mathrm{P}<0.01)$ and no change in interpeak latencies between genders in left ear of head phone users

Table 4: Left ear peak and inter-peak latencies and of BERA among male and female head phone user

\begin{tabular}{|l|c|c|c|}
\hline PEAK LATENCY $(\mathrm{ms})$ Mean \pm SD & Male & Female & P Value \\
\hline I & $1.63 \pm 0.25$ & $1.78 \pm 0.18$ & 0.22 \\
\hline II & $2.48 \pm 0.28$ & $2.37 \pm 0.32$ & 0.54 \\
\hline III & $3.49 \pm 0.31$ & $3.64 \pm 0.27$ & 0.35 \\
\hline IV & $4.84 \pm 0.32$ & $4.63 \pm 0.41$ & 0.33 \\
\hline V & $5.26 \pm 0.34$ & $5.41 \pm 0.28$ & 0.40 \\
\hline
\end{tabular}




\begin{tabular}{|l|c|c|c|}
\hline INTERPEAK LATENCY(ms) Mean \pm SD & Male & Female & P Value \\
\hline I-III & $1.85 \pm 0.46$ & $1.86 \pm 0.31$ & 0.98 \\
\hline I-V & $3.63 \pm 0.53$ & $3.63 \pm 0.28$ & 0.99 \\
\hline III-V & $1.77 \pm 0.11$ & $1.77 \pm 0.39$ & 0.99 \\
\hline
\end{tabular}

Table 4 shows that there was no significant difference in peak latencies of waves I to $\mathrm{V}$ and interpeak latencies between genders in left ear of head phone users.

\section{Discussion}

The mobile phones have become indispensable as communication tools; however there is only limited information exists about the interaction between headphone usage and cognitive functions. Studies showed that chronic exposure to sound more than $90 \mathrm{~d} \mathrm{~B}$ can result in permanent hearing loss ${ }^{6}$ an MP3 player can effectively produce a sound of $120 \mathrm{~d} \mathrm{~B}$, which is further amplified as much as 6-9 d B, while it is being used with an earphone. Thus, listening to louder sounds over long term produce hearing loss. The cell phone music has become one of the most common devices for listening to music in adults and teens. The students very often put on their ear phone and listen to music whenever they get time and usage has become more rampant.

Hearing loss is often thought of as a natural by product of aging process. However, studies are beginning to show that hearing loss is becoming increasingly more common amongst younger people, which is due to chronic exposure to loud noise above $90 \mathrm{~d} \mathrm{~B}$, according to the National Institute on Deafness and other Communication Disorders. Additionally the use of ear buds (earphones) placed directly into the ear can amplify the sound signal by as much as $6-9 \mathrm{~d}$ B. The enhanced sound quality, convenience and portability translate into more time spent listening to music and consequently increased the potential for hearing loss.

In our study, the peak latencies of waves I to $\mathrm{V}$ have remained same between headphone users of both genders. The wave I also provides valuable real time information regarding blood flow to cochlea ${ }^{7}$. The increased latency wave $\mathrm{V}$ might be due to the damage to the inner hair cells. The statistically insignificant variations $(\mathrm{P}>0.05)$ in inter-peak latencies I-III, I-V and III-V in both the ears which have been discussed above, can be explained to be due to an overall prolongation of the absolute latencies of the waves I, III, V. In the present study, as the I-V latency remained normal which shows the integrity of VIII cranial nerve.

On the contrary, a study done by Mazlan et $\mathrm{al}^{8}$ to evaluate the ear infection and hearing loss amongst headphone users, there was no significant association between hearing loss and exposure to sound from headphone usage. In our study, there was no significant difference in peak and inter-peak latencies of the BERA waves between the genders, except the peak latency of wave $\mathrm{V}$ in the left ear of headphone users. This finding was expected since the sounds from the headphones are of low intensity. The average measurement of sound intensity from the headphones was found to be $58 \mathrm{~d}$ B. It was generally below $85 \mathrm{~d} \mathrm{~B}$, which was the threshold above which prolonged exposure of music for 8 hours or more which may cause a permanent hearing loss.

\section{Conclusion}

Thus, we conclude from our observation that the peak latency wave $\mathrm{V}$ has shown a significant difference between genders, with latency being more in males when compared to females. This study may help in the objective assessment of the amount of hearing loss that is to occur on prolonged exposure to the electromagnetic waves and the loud noise emitted by the headphones. The student population needs awareness and understanding of the harmful nature of prolonged usage of earphone music. In future, this may emerge as a risk factor for hearing loss and reduced cognitive functions which may be noise induced/electromagnetic radiation induced.

Acknowledgment: The financial support given by Indian Council of Medical Research is greatly acknowledged.

\section{Conflict of Interest: Nil.}

\section{References}

1. Alice Park. IPod safety: Preventing Hearing loss in teens - Available from: TIME.2009.content.time. com/time/health/article/0,8599,1881130,00.html [Last accessed on: 2015, October 05]. 
2. Oftedal G, Wilen J, Sandstrom M, Mild KH. Symptoms experienced in connection with mobile phone use. Occup Med 2000; 50(4): 237-45.

3. Zhao F, Manchaiah VK, French D, Price SM. Music exposure and hearing disorders: an overview. Int $\mathrm{J}$ Audiol 2010; 49(1): 54-64.

4. Jewett DL, Williston JS. Auditory-evoked far fields averaged from the scalp of humans. Brain. 1971; 94(4): 681-696.

5. Maria Khatoon, Sunita Nighute and Mohammed Ishaque. Effect of gender on brain stem auditory evoked potential. Journal of Recent Advances in Applied Sciences 2013; 28: 113-117.
6. Tay T, Wang JJ, Kifley A, Lindley R, Newall P and Mitchell P. Sensory and cognitive association in older persons: findings from an older Australian population. Gerontology 2006; 52(6): 386-94.

7. Neil Bhattacharyya. Auditory Brainstem Response Audiometry. Medscape. Available from: emedicine.medscape.com/article/836277-overview [Last accessed on: 2015, October 05].

8. R. Mazlan, L. Saim, A. Thomas, R. Said and B. Liyab. Ear infection and hearing loss amongst headphone users. Malays J Med Sci 2002; 9(2): 17-22. 